\title{
Response to acute concussive injury in soccer players: is gender a modifying factor?
}

\author{
Clinical article
}

\author{
Scott L. Zuckerman, M.D., ${ }^{1}$ Gary S. Solomon, Ph.D., ${ }^{2}$ Jonathan A. Forbes, M.D., ${ }^{4}$ \\ Richard F. HaAse, Ph.D., ${ }^{3}$ Allen K. Sills, M.D., ${ }^{4}$ and Mark R. Lovell, Ph.D. ${ }^{5}$ \\ ${ }^{l}$ Vanderbilt University School of Medicine; ${ }^{2}$ Departments of Psychiatry and Neurological Surgery, \\ Vanderbilt Sports Concussion Center, Vanderbilt University School of Medicine; and ${ }^{4}$ Department of \\ Neurological Surgery, Vanderbilt Sports Concussion Center, Nashville, Tennessee; ${ }^{3}$ Department of \\ Educational and Counseling Psychology, University at Albany, State University of New York, Albany, \\ New York; and ${ }^{5}$ ImPACT Applications, Pittsburgh, Pennsylvania
}

\begin{abstract}
Object. Several studies have suggested a gender difference in response to sports-related concussion (SRC). The Concussion in Sport group did not include gender as a modifying factor in SRC, concluding that the evidence at that point was equivocal. In the present study the authors endeavored to assess acute neurocognitive and symptom responses to an SRC in equivalent cohorts of male and female soccer players. The authors hypothesized that female athletes would experience greater levels of acute symptoms and neurocognitive impairment than males.

Methods. Baseline symptom and neurocognitive scores were determined in 40 male and 40 female soccer players by using the Immediate Postconcussion Assessment and Cognitive Testing (ImPACT) scale prior to any SRC. After sustaining an SRC, each athlete completed postconcussion ImPACT tests and was carefully matched on a wide array of biopsychosocial variables. Baseline symptom and neurocognitive test scores were compared, and their acute symptoms and neurocognitive responses to concussive injury were assessed.

Results. Specific a priori hypotheses about differences between males and females at baseline and at postconcussion measurements of verbal and visual memory ImPACT scores were evaluated according to simple main effects of the gender variable and according to baseline-to-postconcussion main effect and interaction of $2 \times 2$ split-plot ANOVA. Neither the interaction nor the main effects nor the simple main effects for either ImPACT variable were found to be statistically significant. Exploratory ANOVAs applied to the remaining ImPACT variables of visualmotor speed, reaction time, impulse control, and symptom total scores revealed only a single statistically significant baseline-to-postconcussion main effect for the symptom total.

Conclusions. The results failed to replicate prior findings of gender-specific baseline neurocognitive differences in verbal and visual memory. The findings also indicated no differential gender-based acute response to concussion (symptoms or neurocognitive scores) among high school soccer players. The implications of these findings for the inclusion of gender as a modifying factor in this tightly matched cohort are addressed. Potential explanations for the null findings are discussed.
\end{abstract}

(http://thejns.org/doi/abs/10.3171/2012.8.PEDS12139)

KEY Words $\bullet \quad$ sports-related concussion $\bullet \quad$ gender $\bullet \quad$ soccer $\bullet \quad$ trauma $\bullet$
mild traumatic brain injury $\bullet \quad$ Immediate Postconcussion Assessment and Cognitive Testing

I $\mathrm{N}$ the US, 1.7-3.8 million TBIs occur each year, and more than 300,000 are due to sports and recreational activities. ${ }^{11,14}$ During the last decade, emergency department visits for sports-related TBIs among children and adolescents increased by $60 \% .{ }^{11}$ Furthermore, SRCs

\footnotetext{
Abbreviations used in this paper: $\mathrm{ADHD}=$ attention deficit hyperactivity disorder; CIS = Concussion in Sport; ImPACT = Immediate Postconcussion Assessment and Cognitive Testing; NCAA $=$ National Collegiate Athletic Association; SRC $=$ sportsrelated concussion; $\mathrm{TBI}=$ traumatic brain injury.
}

comprise nearly $9 \%$ of all injuries sustained during athletic endeavors $;{ }^{13} \mathrm{SRCs}$ represent a growing problem that affects athletes at the junior high and high school, collegiate, professional, and recreational levels.

Since Title IX was passed in 1972, women's involvement in sports has grown exponentially. Soccer has consistently emerged as one of the most popular sports among female athletes in recent decades. From 1990 to 2010, the number of female high school athletes increased from 1.9 million to 3.2 million (http://www.nfhs.org/Participation/ HistoricalSearch.aspx). In the same 20-year time period, 
participation in high school girls' soccer has more than tripled, jumping from 111,711 to 356,116 participants, and the number of NCAA schools participating in women's soccer has increased by 617 programs, the most of any sport played by collegiate women (http://www.nfhs.org/ Participation/HistoricalSearch.aspx). ${ }^{27}$

As female participation in sports has increased, the role of gender in SRC has emerged as a crucial area of study. Several studies have indicated gender differences with respect to SRC incidence, ${ }^{8,13,15,28}$ symptom reporting, $2,5,7$, $8,20,29,31$ and neurocognitive testing, $2,5,6$ both in the baseline $1,3,7,34$ and acute, postconcussion phases.

In 2008, the third meeting of the CIS group was held in Zürich. ${ }^{25}$ The CIS group delineated several "modifying" factors that influence investigation and management of concussions and prolonged symptoms, such as the number of previous concussions, prolonged loss of consciousness, age, medications, and others. However, despite considerable debate, the international group concluded: "There was not unanimous agreement that the current published research evidence is conclusive that this should be included as a modifying factor, although it was accepted that gender may be a risk factor for injury and/ or influence injury severity."

The controversy regarding gender differences in baseline assessment and acute postconcussion outcome has sparked a strong need for empirical study in an area with few definitive answers. More importantly, definitive gender-based data are needed to manage athletes safely when making return-to-play decisions.

Although males overall have higher numbers of participants in sports, females have been shown to have a higher incidence rate of SRC. ${ }^{8,13,15,28}$ At the high school level, Powell and Barber-Foss ${ }^{28}$ observed that participants in girls' soccer, basketball, and softball had a higher concussion rate than those in the equivalent boys' sports. Furthermore, Gessel et al. ${ }^{13}$ also found that concussions accounted for a higher game injury rate in high school girls' sports than in boys' sports. Similarly, at the collegiate level, Hootman et al. ${ }^{15}$ analyzed NCAA injury data and found that females, when compared with males, had a higher concussion rate in the sports of ice hockey, soccer, and basketball. Moreover, Covassin et al. ${ }^{8}$ found that collegiate female athletes were at a higher risk of ingame concussions while playing soccer and basketball compared with males playing the same sports. However, despite concrete incidence data, gender differences in concussion outcomes have not been well described, and our information on symptoms and neurocognitive testing remains largely anecdotal. ${ }^{12}$

Studies also have revealed baseline gender differences in symptoms reported and in neurocognitive test scores. In terms of baseline symptom reporting, Covassin et al. ${ }^{7}$ found small but significant gender differences at baseline, with females out-reporting males on the symptoms of headache, fatigue, sleep difficulty, irritability, sadness, nervousness, feeling more emotional, feeling slowed down, and difficulty concentrating.

In the acute postconcussive state, females have been hypothesized and shown to report increased symptoms compared with their male counterparts..$^{2,5,6,10,20,27,31}$ Colvin et al. ${ }^{5}$ and Broshek et al. ${ }^{2}$ tested male and female athletes in the acute postconcussive state. In both cohorts, women reported a higher number of symptoms than men. Preiss-Farzanegan et al. ${ }^{29}$ assessed 260 adult and pediatric athletes who presented to the emergency department with an SRC and found that women specifically reported higher rates of headache, dizziness, fatigue, and concentration problems compared with male patients. Furthermore, Covassin et al. ${ }^{6}$ evaluated 41 collegiate male and 38 collegiate female athletes and found that men reported postconcussion vomiting and sadness more often than women. However, these authors did not find gender-based symptom-reporting differences in the acute postconcussive phase.

The gender trends reported in the SRC literature appear to be similar to the data reported in members of the general population who sustain a concussive injury. In a meta-analysis of 8 studies, Farace and Alves ${ }^{10}$ concluded that TBI outcomes in the general population were worse for women than men in $85 \%$ of somatic symptoms, including memory, dizziness, fatigue, sensitivity to light and noise, concentration, headache, anxiety, and depression.

Gender differences in baseline neurocognitive functioning in athletic populations have also been found. . $3,7,34^{-1}$ A trend has been found wherein women perform better on verbal memory tasks whereas men perform better on visual memory tasks. For example, Weiss et al. ${ }^{34}$ examined 51 female and 46 male college students and found this trend, with women scoring higher on verbal memory and men performing better on visuospatial tasks, although the authors noted that the effect sizes were small, with the amount of overlap greatly outweighing any differences. Covassin et al. ${ }^{7}$ also found the same trend in an investigation conducted in more than 1200 collegiate athletes, with women scoring higher on verbal memory tasks, and men being more proficient with visual memory.

Gender-based differences in postconcussion neurocognitive scores also have been reported. Colvin et al. ${ }^{5}$ assessed 141 female and 93 male soccer athletes in the postconcussive state, and found that females had significantly slower reaction times than males. In addition, a nonsignificant trend toward poorer female performance on general memory and visual-motor processing speed was found. However, in this study, athletes were tested at different times postinjury and no preinjury data were available. Broshek et al. ${ }^{2}$ administered neurocognitive tests to a mixed group of 37 female and 94 male collegiate athletes in the acute, postconcussive state and compared results with baseline examinations. Following SRC, females had significantly greater declines in simple and complex reaction times compared with preseason baseline levels than did their male counterparts. From a methodological perspective, however, this study included athletes from different sports, did not control for athletes' concussion history, and enlisted nearly 3 times as many males as females. Furthermore, females were an average of 1.7 years younger than males and underwent testing an average of 1 day after males. Finally, Covassin et al. ${ }^{6}$ evaluated a mixed group of 38 female and 41 male collegiate athletes and identified gender differences in 1 of 5 cognitive domains in the acute postconcussive state. Spe- 


\section{S. L. Zuckerman et al.}

cifically, concussed female athletes demonstrated significantly lower visual memory scores than males.

Based on these prior studies, gender differences in athletes at baseline and in the acute postconcussive phase is an active area of research, but remains somewhat murky, with general trends but few definitive answers. The controversy regarding gender differences in acute postconcussion outcomes has sparked a strong need for sound research. Furthermore, definitive data are needed to manage male and female athletes safely when they return to play after a concussion.

To our knowledge, no study has collected baseline and postconcussion ImPACT scores in a cohort of sportspecific athletes matched by age, education level, concussion history, and time of postconcussion testing. We endeavored to assess acute neurocognitive and symptom responses to an SRC in a demographically homogeneous cohort of male and female soccer players. Based on the prior literature, we hypothesized that female soccer athletes, when compared with male soccer athletes, would fit the following patterns: A) they would report more symptoms at baseline and postconcussion; B) they would have better verbal memory scores at baseline; C) they would have poorer baseline visual memory scores; and D) they would experience greater levels of acute, postconcussive neurocognitive impairment.

\section{Methods}

Institutional review board approval was obtained for this study, and all participants provided written, informed consent.

\section{Patient Population}

The initial patient pool contained athletes in archival databases located in the greater middle Tennessee and western Pennsylvania areas who had sustained concussions during competitive soccer participation. The inclusion criteria for this study were as follows: 1) concussion sustained while playing soccer; 2 ) the availability of valid baseline and postconcussion symptom and neurocognitive test data; 3) postconcussion testing within 10 days of injury; and 4) English as the primary language. Following head injuries, concussion was diagnosed based on the on-field presentation of one or more of the following signs or symptoms: 1) self-reported postconcussive symptoms such as lethargy, fogginess, headache; 2) alteration in mental status; 3 ) loss of consciousness; and 4) amnesia. The initial concussion diagnosis was made by a sports medicine athletic trainer or a team physician of one of the competing teams.

We chose to use ImPACT ${ }^{23}$ (a commercially available computerized platform for SRC) for the assessment of symptoms and neurocognitive functions. The ImPACT evaluation includes the revised 22-item Postconcussion Symptom Scale $^{22}$ and 6 neurocognitive tests yielding composite indices of verbal memory, visual memory, visual-motor (processing) speed, reaction time, and impulse control. Studies have shown ImPACT to be reliable $9,18,26,32$ and valid ${ }^{16,17,19,33}$ in the assessment of SRC. Exclusionary criteria were as follows: 1) invalid baseline or postconcus- sion neurocognitive test scores (defined operationally as an ImPACT Impulse Control Composite score of > 30);21 2) self-reported history of special education, speech therapy, repeated year(s) of school, learning disability, ADHD, dyslexia, or autism; 3) self-reported history of brain surgery or seizure disorder; and 4) self-reported history of treatment for drug/alcohol abuse or psychiatric illness.

Data extracted from each deidentified record included athlete-reported sex, age, height, weight, years of education, concussion history, and neuropsychiatric history. Dates of baseline testing, concussion, and posttesting were recorded, which allowed for the calculation of days elapsed between concussion and postinjury testing. Raw ImPACT scores were recorded for the composites of verbal memory, visual memory, visual-motor (processing) speed, reaction time, impulse control, and total symptoms. In addition, baseline and postconcussion values of all 22 symptoms were detailed.

\section{Statistical Analysis}

Statistical analyses consisted of both descriptive and inferential procedures. The success of the matching process was evaluated by t-tests of male-female differences on 13 inclusion and exclusion criteria. The means and SDs of the ImPACT dependent variables for both males and females were assessed at both baseline and postconcussion measurements. Tests of the a priori Hypotheses A-D about gender differences on the ImPACT dependent variables were initially evaluated by a 2 (gender) $x$ 2 (baseline, postconcussion) split-plot ANOVA with repeated measures on the baseline-to-postconcussion factor. ${ }^{24}$ Omnibus test statistics $(F)$ were evaluated for the main effects of gender and of baseline-to-postconcussion as well as for the gender by baseline-to-postconcussion interaction. Simple main effects were evaluated for each of the a priori hypotheses at $\alpha=0.05$. Tests of exploratory post hoc hypotheses were evaluated by a Bonferroni adjusted $\alpha=0.05 / 4=0.0125$. All analyses were performed using SPSS and STATA software.

\section{Results}

\section{Matching of Male and Female Samples}

Our study included a total of 40 male and 40 female soccer players. Comparisons of the inclusion and exclusion criteria variables revealed that the group-matching process was successful in achieving group distribution equality for the male and female samples. The male and female groups were matched identically on history of special education, speech therapy, year(s) of school repeated, learning disability, ADHD, dyslexia, autism, history of brain surgery or seizure disorder, or history of treatment for drug/alcohol abuse. There were no occurrences $(0$ of $15)$ of any of these variables in either group.

Males and females also had nearly equal numbers of prior concussions $(0.68 \pm 1.12$ vs $0.65 \pm 1.05$ concussions, respectively [mean $\pm \mathrm{SD}])$ and thus did not differ $(t(78)$ $=0.85, \mathrm{p}=0.92)$. The number of years of education was slightly higher for females $(9.68 \pm 1.59$ years $)$ than for males $(9.53 \pm 1.62$ years $)$, but the means did not differ 
significantly $(t(78)=0.42, \mathrm{p}=0.68)$. The females were older than the males by approximately 1 month $(15.9 \pm$ 1.75 years vs $15.8 \pm 1.88$ years, respectively), but the difference was both negligible and statistically insignificant $(t(78)=0.25, \mathrm{p}=0.81)$. One female $(2.5 \%)$ and no males reported past psychiatric treatment, but the difference is not statistically significant $(t(77)=0.99, \mathrm{p}=0.33)$. Finally, the females were tested postconcussion approximately 2 days later than the males $(7.18 \pm 6.35$ days vs $5.40 \pm 4.44$ days), but the mean number of days did not differ statistically $(t(78)=1.45, \mathrm{p}=0.15)$. The matching process was successful in all of those variables that were considered to be potential confounders to any observed male-female difference in the neurocognitive dependent variables.

\section{Tests of A Priori Hypotheses and Post Hoc Exploratory} Analyses

Tests of hypotheses were performed on 2 classes of variables: 1) those for which specific a priori hypotheses were articulated; and 2) exploratory $2 \times 2$ split-plot ANOVAs for which we had no specific a priori predictions. Split-plot ANOVA designs are factorial designs that consist of both between-group (for example, males vs females) and within-subject factors that consist of repeated observations on the same cases (that is, pre- and posttest assessments). Within-subject factors are typically more powerful than between-group factors because they use each individual as his/her own control.

The specific a priori hypotheses of this study focused on the verbal and visual memory scores of the ImPACT battery. The means and SDs for male and female respondents for these 2 measures are displayed in Table 1 . The results for the 2 main effects and the interaction of the 2 (gender) $\times 2$ (baseline, postconcussion) split-plot ANOVA are summarized in Table 2 . The results of the simple main effects pertaining to the hypotheses are summarized in the text. Hypotheses regarding the 2 memory variables were evaluated for statistical significance at $\alpha=0.05$ without further adjustment insofar as these hypotheses are a priori in nature. ${ }^{30}$

The remaining 4 variables of the ImPACT battery (visual-motor speed, reaction time, impulse control, and total symptoms), although not specifically hypothesized a priori, may nonetheless have informative exploratory value. The male and female means and SDs are summarized in Table 1, and the results of the $2 \times 2$ split-plot ANOVAs are shown in the lower part of Table 2 . Simple main effects were computed only following a significant interaction; conditioning the follow-up test on a significant interaction constitutes a Fisher least significance test control for escalating Type I error rates. ${ }^{30}$ Because of the exploratory nature of tests in 4 of the 6 ImPACT variables, post hoc hypotheses were evaluated for significance at a more conservative Bonferroni adjusted $\alpha=0.05 / 4=0.0125$. The interpretation of statistical significance for these post hoc explorations should be cautiously evaluated.

\section{A Priori Hypothesis Tests of Verbal and Visual Memory}

The results for the main and interaction effects of the $2 \times 2$ split-plot ANOVA on verbal and visual memory are
TABLE 1: Baseline and postconcussion measurements of ImPACT variables in male and female high school soccer players

\begin{tabular}{lrrrrr}
\hline & \multicolumn{2}{c}{ Male Patients } & & \multicolumn{2}{c}{ Female Patients } \\
\cline { 2 - 3 } \cline { 5 - 6 } Measure & Mean & SD & & Mean & SD \\
\hline baseline score & & & & \\
verbal memory & 82.47 & 8.20 & & 84.55 & 9.95 \\
visual memory & 76.80 & 11.55 & & 72.00 & 13.49 \\
visual-motor speed & 38.19 & 6.22 & & 37.67 & 5.96 \\
reaction time & 0.61 & 0.14 & & 0.58 & 0.07 \\
impulse control & 6.62 & 7.57 & 6.50 & 3.65 \\
symptom total & 2.03 & 2.75 & 3.95 & 5.87 \\
postconcussion score & & & & \\
verbal memory & 83.60 & 13.10 & & 82.15 & 15.03 \\
visual memory & 70.58 & 15.52 & & 68.80 & 16.28 \\
visual-motor speed & 38.12 & 8.91 & & 37.01 & 8.43 \\
reaction time & 0.59 & 0.09 & & 0.61 & 0.13 \\
impulse control & 7.70 & 8.43 & 8.48 & 5.96 \\
symptom total & 12.75 & 21.75 & 13.18 & 16.05 \\
\hline
\end{tabular}

summarized in Table 2. The main effects are not especially interesting because the gender effect is the average of baseline and postconcussion scores, and the baselineto-postconcussion effect is averaged over male and female participants. The interaction effect, however, is quite meaningful in that it evaluates the difference between males and females on the mean change from baseline to postconcussion, or conversely (because interactions are symmetrical) it evaluates the mean difference between males and females at baseline versus postconcussion. The means and SDs of the male and female samples at baseline and postconcussion are shown in Table 1. There are no statistically significant interaction effects for either verbal or visual memory (see Table 2). The a priori test of the simple main effect of males versus females at baseline showed no significant differences for verbal memory $(t(78)=1.02, \mathrm{p}=0.312)$ or for visual memory $(t(78)=$ $-1.71, \mathrm{p}=0.091)$. Similarly, there were no significant simple gender main effects at postconcussion measurement for verbal memory $(t(78)=-0.46, \mathrm{p}=0.671)$ or for visual memory $(t(78)=-0.50, \mathrm{p}=0.619)$. Hence Hypotheses A, $\mathrm{B}$, and $\mathrm{C}$ could not be verified. The standardized mean difference effect sizes of these simple main effects $(0.10$ $\leq \hat{\delta} \leq 0.387$ ) are consistent with the Cohen ${ }^{4,5}$ definition of a small to small or medium effect.

Post Hoc Tests of Visual-Motor Speed, Reaction Time, and Total Symptoms

The main and interaction effects of the $2 \times 2$ splitplot ANOVA on the remaining ImPACT variables are presented in the last 4 rows of Table 2. The means and SDs for these variables appear in Table 1. The mean baseline (2.99) versus postconcussion (12.97) main effect for total symptoms proved to be statistically significant $(F(1$, $78)=21.60, \mathrm{p}<0.001)$. The baseline $(6.56)$ versus postconcussion (8.09) mean difference for impulse control was not found to be a statistically significant difference 


\section{S. L. Zuckerman et al.}

TABLE 2: Gender effects assessed using baseline-to-postconcussion split-plot ANOVAs in high school soccer players*

\begin{tabular}{|c|c|c|c|c|c|c|}
\hline \multirow[b]{2}{*}{ Variable } & \multicolumn{2}{|c|}{ Gender Main Effect } & \multicolumn{2}{|c|}{ Baseline-to-Postconcussion Main Effect } & \multicolumn{2}{|c|}{ Interaction Effect } \\
\hline & $\mathrm{F}$ & $p$ Value & $\mathrm{F}$ & $p$ Value & $\mathrm{F}$ & $\mathrm{p}$ Value \\
\hline \multicolumn{7}{|l|}{ a priori tests } \\
\hline verbal memory & 0.293 & 0.590 & 0.176 & 0.676 & 1.345 & 0.250 \\
\hline visual memory & 1.448 & 0.233 & 7.936 & 0.06 & 0.818 & 0.369 \\
\hline \multicolumn{7}{|l|}{ post hoc tests } \\
\hline visual-motor speed & 0.293 & 0.590 & 0.247 & 0.620 & 0.165 & 0.685 \\
\hline reaction time & 0.072 & 0.790 & 0.074 & 0.786 & 5.397 & 0.023 \\
\hline impulse control & 0.055 & 0.815 & 7.687 & 0.07 & 0.669 & 0.416 \\
\hline symptom total & 0.274 & 0.602 & 21.597 & $<0.001$ & 0.122 & 0.728 \\
\hline
\end{tabular}

* Degrees of freedom for effect and error are 1 and 78 , respectively, for all tests. The critical value of $\alpha$ for declaring significance of the a priori tests is $\alpha=0.05$, and the Bonferroni adjusted critical value of $\alpha$ for post hoc tests is $\alpha=0.05 / 4=0.0125$. Abbreviation: $\mathrm{F}=$ variance of the group means/the mean of the within-group variances.

$(F(1,78)=7.69, \mathrm{p}=0.07)$. No other main effects or any of the interactions in the set of post hoc exploratory analyses were found to be statistically reliable because they did not exceed the Bonferroni adjusted $\alpha=0.0125$ criterion.

In summary, we could not confirm our predictions of differences between male and female soccer players on either of the hypothesized memory variables. Although 2 of the baseline versus postconcussion main effects proved to be statistically significant differences, none of the interactions measuring change conditioned on gender reached statistical significance.

\section{Discussion}

The CIS group did not include gender as a risk factor in concussion, but has stated that gender "may be a risk factor for injury and/or influence injury severity." ${ }^{25} \mathrm{We}$ assessed the acute response to concussion in a homogeneous group of high school soccer players in an attempt to shed some empirical light on the potential role of gender as a modifying factor. Prior studies have used mixed groups of high school and collegiate athletes, and have not routinely and consistently controlled for the potentially confounding variables of age, years of education, specific sport, number of prior concussions, time of testing postinjury, medical history, and other biopsychosocial moderating variables (for example, medical history, presence of ADHD and/or learning disability, and so on). We assessed the acute neurocognitive and symptom responses to concussive injury in a group of 40 males and 40 females, all of whom were matched closely on the above-noted variables.

We were unable to replicate the previously reported gender differences in symptoms at baseline $e^{1,3,7,34}$ or in the immediate postconcussion phase. . $^{2,5,6,10,20,29,31}$ Several possible explanations exist. First, in the current study we assessed total symptom score and did not compare groups on individual symptoms. This was done initially in an attempt to minimize the potential experiment-wise error rate. Prior studies have described differences in genderbased reporting of individual symptoms ${ }^{6,29}$ as opposed to a symptom score total..$^{2,47}$ Some inconsistency has also been noted in gender-related total symptom scores postconcussion. ${ }^{6}$ Second, inspection of the mean postconcussion symptom scores (Table 2) shows no significance between groups, but extremely large SDs, suggesting dramatic across-group variability in symptom reporting. Additionally, it is possible that our sample size of 80 had insufficient power to detect any differences in total symptom scores; previous studies have included as many as 260 patients. We were able to assess only 80 athletes in this study due to the inherent limitations of our stringent matching criteria. Finally, it is possible that prior studies have shown differential gender-based symptom reporting due to the mixing of athletes from a multitude of sports, with various concussion histories, and with different times of testing postconcussion.

We were unable to replicate the previously reported baseline differences between the sexes on neurocognitive measures of verbal and visual memory. Historically, males have outperformed females on visual memory tests, whereas the converse has been the case with verbal memory. ${ }^{1,3,7,34}$ This could have been due to our smaller sample size, because a study of 97 patients found this effect marginally, ${ }^{34}$ whereas a study assessing more than 1200 patients $^{7}$ found a definite gender effect. Again, the role of the specific sport may be a relevant factor, because previous studies have assessed athletes from a variety of sports, whereas the current study assessed soccer players only.

Finally, we found no significant gender effect on postconcussion neurocognitive scores. Prior research has shown slower reaction time scores in females postconcussion, ${ }^{2,5}$ but the first study did not control for time of testing postinjury and the second study did not control for concussion history. Both studies used athletes from a variety of sports.

Our study does suffer from limitations. First, this was a cross-sectional, retrospective study and was not conducted prospectively. Second, our sample size of 80 patients may have been lacking in power to detect some of the symptom and neurocognitive effects hypothesized and noted in prior research. As mentioned previously, 
however, the rigorous matching criteria used in this study significantly reduced the number of patients available for inclusion. Third, we did not assess for gender-based differences in specific concussion symptoms as some studies have done, but chose instead to evaluate the total symptom score. We chose this approach to focus on the major hypotheses of the study and to minimize the potential inflation of the experiment-wise error rate, which would have been incurred by performing another 22 statistical comparisons. Furthermore, we did not control for injury severity. Finally, we did not assess athletes formally for effort by using traditional clinical neuropsychological tests. Instead, we used the rule proposed by Lovell as a proxy for a formal effort test.

Conversely, the study does have its strengths. The rigorous matching criteria used created groups that accounted for a variety of factors that have been posited to be relevant in concussion research. This study took into account neuropsychiatric, demographic, biopsychosocial, and concussion-relevant parameters and factors in its experimental design in an effort to match groups as closely as possible. We used reasonable statistical procedures for data analysis, and set conservative levels of significance for exploratory hypotheses.

\section{Conclusions}

We urge future researchers to attempt to use sportspecific populations in concussion studies because it is quite possible that there are sport-specific responses to concussive injury in symptoms and neurocognitive scores. The results of this study could well be interpreted to indicate that there is no gender-based difference in acute response (symptoms or neurocognitive scores) to concussive injury in high school athletes. As an interim position, we view the results of our study as supportive of the current CIS group's position regarding the role of gender as a modifying factor in SRC, at least for athletes in soccer.

\section{Disclosure}

Dr. Lovell owns stock in ImPACT Applications. The authors report no conflict of interest concerning the materials or methods used in this study or the findings specified in this paper.

Author contributions to the study and manuscript preparation include the following. Conception and design: Solomon, Sills, Lovell. Acquisition of data: Solomon, Sills, Lovell. Analysis and interpretation of data: Zuckerman, Solomon, Forbes, Haase. Drafting the article: Zuckerman, Solomon, Haase. Critically revising the article: all authors. Reviewed submitted version of manuscript: all authors. Approved the final version of the manuscript on behalf of all authors: Zuckerman. Statistical analysis: Solomon, Haase. Administrative/technical/material support: Solomon, Sills, Lovell. Study supervision: Solomon, Sills, Lovell.

\section{References}

1. Barr WB: Neuropsychological testing of high school athletes. Preliminary norms and test-retest indices. Arch Clin Neuropsychol 18:91-101, 2003

2. Broshek DK, Kaushik T, Freeman JR, Erlanger D, Webbe F, Barth JT: Sex differences in outcome following sports-related concussion. J Neurosurg 102:856-863, 2005

3. Brown CN, Guskiewicz KM, Bleiberg J: Athlete characteristics and outcome scores for computerized neuropsychological assessment: a preliminary analysis. J Athl Train 42:515-523, 2007

4. Cohen J: Statistical Power Analysis for the Behavioral Sciences, ed 2. Hillsdale, NJ: Lawrence Erlbaum Associates, 1988

5. Colvin AC, Mullen J, Lovell MR, West RV, Collins MW, Groh $\mathrm{M}$ : The role of concussion history and gender in recovery from soccer-related concussion. Am J Sports Med 37:16991704,2009

6. Covassin T, Schatz P, Swanik CB: Sex differences in neuropsychological function and post-concussion symptoms of concussed collegiate athletes. Neurosurgery 61:345-351, 2007

7. Covassin T, Swanik CB, Sachs M, Kendrick Z, Schatz P, Zillmer E, et al: Sex differences in baseline neuropsychological function and concussion symptoms of collegiate athletes. Br J Sports Med 40:923-927, 2006

8. Covassin T, Swanik CB, Sachs ML: Sex differences and the incidence of concussions among collegiate athletes. J Athl Train 38:238-244, 2003

9. Elbin RJ, Schatz P, Covassin T: One-year test-retest reliability of the online version of ImPACT in high school athletes. Am J Sports Med 39:2319-2324, 2011

10. Farace E, Alves WM: Do women fare worse: a metaanalysis of gender differences in traumatic brain injury outcome. J Neurosurg 93:539-545, 2000

11. Faul M, Xu L, Wald MM, Coronado VG: Traumatic Brain Injury in the United States: Emergency Department Visits, Hospitalizations, and Deaths 2002-2006. Atlanta: Centers for Disease Control and Prevention, National Center for Injury Prevention and Control, 2010 (http://www.cdc.gov/ traumaticbraininjury/pdf/blue_book.pdf) [Accessed August 24, 2012]

12. Frommer LJ, Gurka KK, Cross KM, Ingersoll CD, Comstock RD, Saliba SA: Sex differences in concussion symptoms of high school athletes. J Athl Train 46:76-84, 2011

13. Gessel LM, Fields SK, Collins CL, Dick RW, Comstock RD: Concussions among United States high school and collegiate athletes. J Athl Train 42:495-503, 2007

14. Gilchrist J: Nonfatal traumatic brain injuries from sports and recreation activities-United States, 2001-2005. MMWR Morb Mortal Wkly Rep 56:733-737, 2007

15. Hootman JM, Dick R, Agel J: Epidemiology of collegiate injuries for 15 sports: summary and recommendations for injury prevention initiatives. J Athl Train 42:311-319, 2007

16. Iverson G, Lovell M, Collins M: Validity of IMPACT for measuring the effects of sports-related concussion. Arch Clin Neuropsychol 17:769, 2002 (Abstract)

17. Iverson GL, Gaetz M, Lovell MR, Collins MW: Relation between subjective fogginess and neuropsychological testing following concussion. J Int Neuropsychol Soc 10:904-906, 2004

18. Iverson GL, Lovell MR, Collins MW: Interpreting change on ImPACT following sport concussion. Clin Neuropsychol 17:460-467, 2003

19. Iverson GL, Lovell MR, Collins MW: Validity of ImPACT for measuring processing speed following sports-related concussion. J Clin Exp Neuropsychol 27:683-689, 2005

20. Lidvall HF, Linderoth B, Norlin B: Causes of the post-concussional syndrome. Acta Neurol Scand Suppl 56:3-144, 1974

21. Lovell MR: ImPACT 2007 (6.0) Clinical Interpretation Manual Fall 2007. Pittsburgh: University of Pittsburgh Medical Center (http://www.impacttest.com/pdf/ImPACT_Clinical _Interpretation_Manual.pdf) [Accessed August 23, 2012]

22. Lovell MR, Collins MW: Neuropsychological assessment of the college football player. J Head Trauma Rehabil 13:9-26, 1998

23. Maroon JC, Lovell MR, Norwig J, Podell K, Powell JW, Hartl $\mathrm{R}$ : Cerebral concussion in athletes: evaluation and neuropsychological testing. Neurosurgery 47:659-672, 2000 


\section{S. L. Zuckerman et al.}

24. Maxwell SE, Delaney HD: Designing Experiments and Analyzing Data: A Model Comparison Perspective. Mahwah, NJ: Lawrence Erlbaum Associates, 2004, p 920

25. McCrory P, Meeuwisse W, Johnston K, Dvorak J, Aubry M, Molloy M, et al: Consensus Statement on Concussion in Sport: the 3rd International Conference on Concussion in Sport held in Zurich, November 2008. Br J Sports Med 43 (Suppl 1):i76-i90, 2009

26. Miller JR, Adamson GJ, Pink MM, Sweet JC: Comparison of preseason, midseason, and postseason neurocognitive scores in uninjured collegiate football players. Am J Sports Med 35:1284-1288, 2007

27. National Collegiate Athletic Association: 1981-82-200809 NCAA Sports Sponsorship and Participation Rates Report. Indianapolis: National Collegiate Athletic Association, 2010 (http://www.ncaapublications.com/productdownloads/ PR2010.pdf) [Accessed August 23, 2012]

28. Powell JW, Barber-Foss KD: Traumatic brain injury in high school athletes. JAMA 282:958-963, 1999

29. Preiss-Farzanegan SJ, Chapman B, Wong TM, Wu J, Bazarian JJ: The relationship between gender and postconcussion symptoms after sport-related mild traumatic brain injury. PM R 1:245-253, 2009

30. Rencher AC, Scott DT: Assessing the contribution of individ- ual variables following rejection of a multivariate hypothesis. Commun Statist Simula 19:535-553, 1990

31. Rutherford WH: Sequelae of concussion caused by minor head injuries. Lancet 1:1-4, 1977

32. Schatz P: Long-term test-retest reliability of baseline cognitive assessments using ImPACT. Am J Sports Med 38:47-53, 2010

33. Schatz P, Pardini JE, Lovell MR, Collins MW, Podell K: Sensitivity and specificity of the ImPACT Test Battery for concussion in athletes. Arch Clin Neuropsychol 21:91-99, 2006

34. Weiss E, Kemmler G, Deisenhammer E, Fleischhacker W, Delazer M: Sex differences in cognitive functions. Pers Individ Dif 35:863-875, 2003

Manuscript submitted February 9, 2012.

Accepted August 21, 2012.

Please include this information when citing this paper: published online October 2, 2012; DOI: 10.3171/2012.8.PEDS12139. Address correspondence to: Scott L. Zuckerman, M.D., Vanderbilt University Medical Center, Department of Neurological Surgery, T-4224 Medical Center North, Nashville, Tennessee 372322380. email: scott.zuckerman@vanderbilt.edu. 